QCD Evolution Workshop: From Collinear to Non-Collinear Case International Journal of Modern Physics: Conference Series Vol. 4 (2011) 115-125

(C) World Scientific Publishing Company

DOI: $10.1142 / S 2010194511001620$

\title{
ANALYTIC PROPERTIES OF MULTI-PARTON POLE MATRIX ELEMENTS AND UNIVERSALITY OF FRAGMENTATION FUNCTIONS
}

\author{
LEONARD GAMBERG \\ Division of Science, Penn State University-Berks \\ Reading, Pennsylvania 19610, USA \\ lpg10@psu.edu \\ ASMITA MUKHERJEE \\ Physics Department, Indian Institute of Technology Bombay, \\ Powai, Mumbai 400076, India \\ asmita@phy.iitb.ac.in \\ P. J. MULDERS \\ Department of Physics and Astronomy, VU University \\ NL-1081 HV Amsterdam, the Netherlands \\ mulders@few.vu.nl
}

Gluonic pole matrix elements explain the appearance of single spin asymmetries (SSA) in high-energy scattering processes. They involve a combination of operators which are odd under time reversal (T-odd). Such matrix elements appear in principle both for parton distribution functions and parton fragmentation functions. We show that for parton fragmentation functions these gluonic pole matrix elements vanish as a consequence of the analytic structure of scattering amplitudes in Quantum Chromodynamics. We extend this analysis to the case of multi-partonic pole matrix elements. This result is important in the study of the universality of transverse momentum dependent (TMD) fragmentation functions.

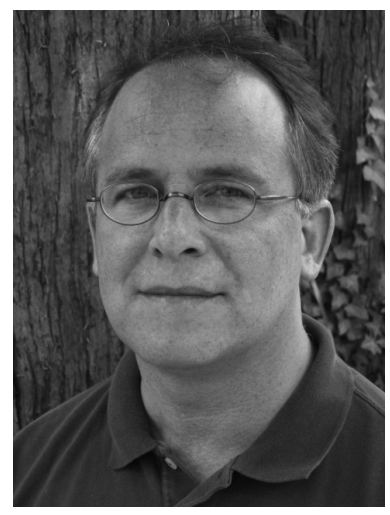




\section{Introduction}

Cross sections for high energy scattering processes are given by a convolution of partonic cross sections with parton distribution functions (PDF) and parton fragmentation functions (PFF). These functions interpreted as momentum distributions and parton decay functions, respectively, are given as matrix elements of quark and gluon operators $[1,2,3,4]$. Among such observables, gluonic pole or Qiu-Sterman matrix elements involve a combination of operators which is odd under time reversal (T-odd). These matrix elements have been extensively studied $[5,6,7,8,9,10,11$, 12]. They explain the appearance of single spin asymmetries (SSA) in high-energy scattering processes. These correlation functions show up in combination with calculable hard parts that may differ from the partonic cross sections through specific calculable factors and signs $[9,13,14]$. In Ref. [15] we use general properties of scattering amplitudes in Quantum Chromodynamics (QCD) to study the support properties of these parton correlation functions. Specifically, assuming unitarity and analyticity properties to hold for forward parton-hadron scattering amplitudes, we uncover their singularity structure which then determines the corresponding properties of the PDFs and PFFs. Using this analysis for fragmentation functions, we show that single gluon and multi-gluon pole matrix elements vanish in the limit when the momenta of these gluons become zero. Since these multi-gluonic pole matrix elements appear in integrated and weighted transverse momentum dependent (TMD) fragmentation functions, as a consequence of them being zero, all leading T-odd effects in the matrix elements are all part of the final state interactions among the fragmentation remnant and final state hadron [16, 4] rather than from T-odd partonic operator combinations. Thus, when transverse momentum dependent (TMD) T-odd fragmentation functions appear in observables like SSA they are convoluted with the standard partonic cross sections. The vanishing of gluonic pole matrix elements for any number of gluons, provides a general proof of the universality of these TMD fragmentation functions [17].

Spectral studies of the gluonic pole matrix elements for fragmentation, specifically for quark-quark-gluon matrix elements with just one gluon field [18, 19] already indicated that they vanish. Our arguments presented here are general and can be applied to multi-gluonic pole matrix elements. They depend only on the analytic structure of scattering amplitudes, yet they do not depend on the details of partonic or hadronic masses, and are insensitive to integrations over transverse momentum.

\section{Correlation Functions as Parton-Hadron Scattering Amplitudes}

We begin by considering high-energy scattering processes where the structure of hadrons is accounted for using quark and gluon correlators, which are Fourier transforms of forward matrix elements of non-local quark and gluon operators between 


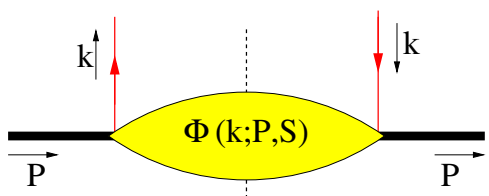

(a)

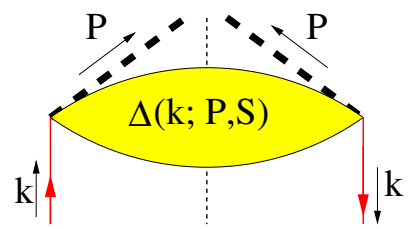

(b)

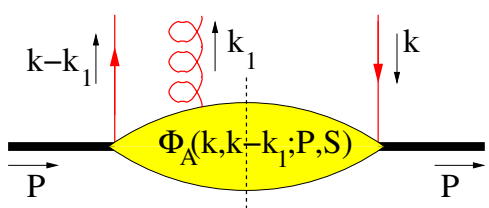

(c)

Fig. 1. The quark-quark correlators that establish the non-perturbative connection between partons and hadrons for distribution functions (a) or fragmentation functions (b) and a quark-quarkgluon (multi-parton) correlator (c).

hadronic states. For instance the quark-quark correlator

$$
\left.\Phi_{i j}^{[\mathcal{U}]}\left(x, k_{T}\right)=\int \frac{d(\xi \cdot P) d^{2} \xi_{T}}{(2 \pi)^{3}} e^{i k \cdot \xi}\left\langle P\left|\bar{\psi}_{j}(0) \mathcal{U}_{[0 ; \xi]} \psi_{i}(\xi)\right| P\right\rangle\right\rfloor_{\mathrm{LF}},
$$

where $\mathcal{U}_{[\eta ; \xi]}=\mathcal{P} \exp \left[-i g \int_{C} d s \cdot A^{a}(s) t^{a}\right]$ is the gauge link that ensures gauge invariance $[20,21,22,23]$. The non-locality of the matrix elements needed to describe the distribution functions is restricted to the light-front (LF) and it is convenient to use the Sudakov decomposition

$$
k=x P+\sigma n+k_{T},
$$

in terms of a generic light-like four-vector $n$ satisfying $n^{2}=0$ and $P \cdot n=1$. In a particular hard process, its role is played by other momenta that are hard with respect to the hadron under consideration, e.g. $n \approx P^{\prime} / P \cdot P^{\prime}$. We can then also work with light-cone coordinates. Including mass effects one would have $n_{-}=n$ and $n_{+}=P-\frac{1}{2} M^{2} n$; with $k^{ \pm} \equiv k \cdot n_{\mp}$. These are $k^{+}=k \cdot n=x$ and $k^{-}=$ $k \cdot P-\frac{1}{2} x M^{2}=\sigma+\frac{1}{2} x M^{2}$. The transverse momentum is orthogonal to $n$ and $P$. In a hard process the dependence on $k^{-}$of a particular correlator is not important and it is integrated over, leaving us with the restricted light-front non-locality $\xi^{+}=0$ (LF). The expansion of these correlators (in Dirac space) contains the TMD distribution functions depending on momentum fraction $x$ and transverse momentum $k_{T}^{2}$. Upon 
integration over $k_{T}$ one obtains the collinear correlators

$$
\left.\Phi(x)=\int \frac{d(\xi \cdot P)}{2 \pi} e^{i x \xi \cdot P}\left\langle P\left|\bar{\psi}(0) \mathcal{U}_{[0 ; \xi]}^{n} \psi(\xi)\right| P\right\rangle\right\rfloor_{\mathrm{LC}},
$$

where non-locality is restricted to the light-cone (LC: $\xi \cdot n=\xi_{T}=0$ ) and the gauge link is unique, being the straight-line path along $n$. These collinear correlators are expanded in the 'standard' parton distribution functions, depending solely on the momentum fraction $x$. Here we will not discuss the scale dependence $[24,25,26$, 27 .

The quark-quark light-front correlator that plays a role in the fragmentation of partons is

$$
\Delta_{i j}^{[\mathcal{U}]}\left(z, k_{T}\right)=\left.\sum_{X} \int \frac{d(\xi \cdot P) d^{2} \xi_{T}}{(2 \pi)^{3}} e^{i k \cdot \xi}\left\langle 0\left|\mathcal{U}_{[0, \xi]} \psi_{i}(\xi)\right| P, X\right\rangle\left\langle P, X\left|\bar{\psi}_{j}(0)\right| 0\right\rangle\right|_{L F},
$$

with the quark momentum, $k=\frac{1}{z} P+k_{T}+\sigma n$, i.e. a Sudakov expansion with $x=1 / z>1$. In this case one often refers to the hadron transverse momentum $P_{\perp}=-z k_{T}$ (in a frame in which the parton does not have a transverse momentum $\left.\left(k_{\perp}=0\right)\right)$. Diagrammatically these correlators are represented in Fig. 1.

Here we we investigate multi-parton correlators by looking at the case with one additional gluon, as given in Fig. 1 (c). They appear in azimuthal asymmetries involving the $k_{T}$-weighted correlator,

$$
\begin{aligned}
\Phi_{\partial}^{\alpha}{ }^{[\mathcal{U}]}(x) & =\int d^{2} k_{T} k_{T}^{\alpha} \Phi^{[\mathcal{U}]}\left(x, k_{T}\right) \\
& =\tilde{\Phi}_{\partial}^{\alpha}(x)+C_{G}^{[\mathcal{U}]} \pi \Phi_{G}^{\alpha}(x, x),
\end{aligned}
$$

which is decomposed in pieces $\tilde{\Phi}_{\partial}$ and $\Phi_{G}$, that contain T-even and T-odd operator combinations, respectively [23]. The T-odd parts come with calculable gluonic pole factors $C_{G}^{[\mathcal{U}]}$ that depend on the gauge link. Here we focus on the connection of this part to the zero momentum $\left(x_{1} \rightarrow 0\right)$ limit of a quark-quark-gluon correlator

$$
\begin{gathered}
\Phi_{G}^{\alpha}\left(x, x-x_{1}\right)=\int \frac{d(\xi \cdot P)}{2 \pi} \frac{d(\eta \cdot P)}{2 \pi} e^{i x_{1}(\eta \cdot P)} e^{i\left(x-x_{1}\right)(\xi \cdot P)} \\
\times\left\langle P\left|\bar{\psi}(0) U_{[0 ; \eta]}^{n} g G^{n \alpha}(\eta) U_{[\eta ; \xi]}^{n} \psi(\xi)\right| P\right\rangle_{L \mathrm{LC}},
\end{gathered}
$$

where $G^{n \alpha}=n_{\mu} G^{\mu \alpha}$ represent specific components of the color field strength tensor ( $\alpha$ being transverse). The zero momentum limit of this correlator is the gluonic pole matrix element mentioned above. It is the support of $\Phi_{G}\left(x, x-x_{1}\right)$ that we are after and specifically the model independent proof that it vanishes in the limit $x_{1} \rightarrow 0$ for the case of the fragmentation correlators $(|x|>1)$.

\section{Analytic Structure of Distribution and Fragmentation Functions}

The first step in these considerations is the observation that the $k^{-}$and $k_{1}^{-}$integrations in the quark-quark and quark-quark-gluon correlators lead to light-front correlators, for which time-ordering is irrelevant. Therefore the matrix elements can 

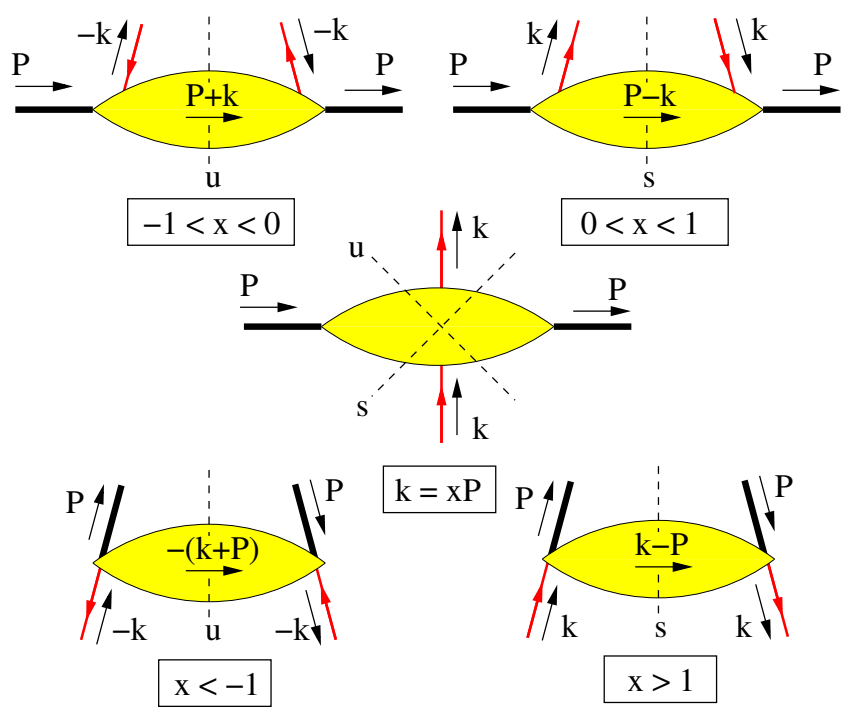

Fig. 2. Integrating parton correlators over $k^{-}$allows connecting them to a single anti-parton hadron scattering four-point function $\mathcal{A}\left(k^{2} ; s, u\right)$ (middle). Depending on the value of $x$, the imaginary part of this amplitude represents the (anti)-parton distribution or fragmentation correlators.

be considered as forward matrix elements of time-ordered products of operators. These represent scattering amplitudes and their analytic structure enables one to make statements about the support of the associated parton correlation functions. This can be done for quark-quark [28] and multi-parton correlators [29] and for TMD as well as collinear correlators [30]. In this language the diagrams of Figs. 1 are just hadron-parton amplitudes, e.g. the quark-quark correlator Eq. (3) related to the forward anti-quark-hadron scattering amplitude $\mathcal{A}\left(k^{2} ; s, u\right)$ (see Fig. 2). Depending on the precise structure these are untruncated Green functions (time-ordered) or related to such Green functions via the LSZ formalism [31].

The second step is the study of the analytic structure of an amplitude and in particular the singularities arising from cuts in the forward amplitudes. These are cuts in the (untruncated) legs, in particular the parton virtuality $k^{2}$, and the Mandelstam invariants. The virtualities are conventionally placed just below the real axis, or the invariants are replaced by $p^{2}+i \epsilon, s+i \epsilon$. The integrations over $k^{-}$ and $k_{1}^{-}$imply integrations over some of the invariants. At this point one must make the standard assumption that it is possible to use analyticity for QCD-amplitudes. We illustrate this step first for the standard quark-quark correlators. In that case one works with the four-point Green functions, shown in the middle of Fig. 2, where the cut amplitude depending on the particular value of $x$ gives the quark distributions $(0<x<1)$ or the quark fragmentation functions $(0<z<1$ or $x>1)$ while analytic continuation to negative $x$ describes the anti-quark distributions $(-1<x<0)$ and anti-quark fragmentation $(-1<z<0$ or $x<-1)$. 


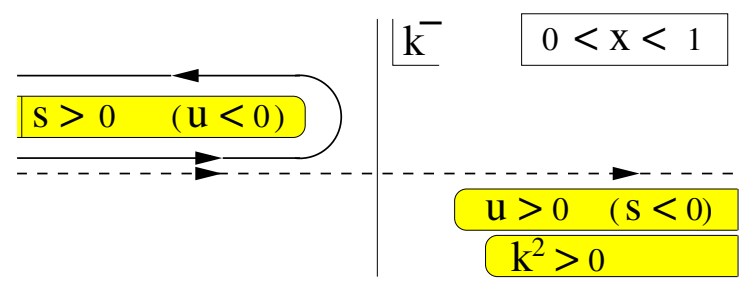

(a) quarks

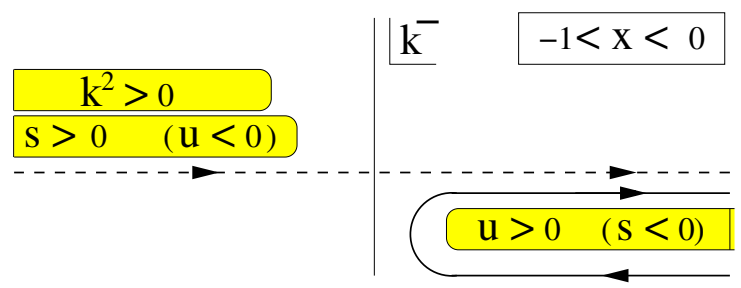

(b) antiquarks

Fig. 3. The integration contours for the $k^{-}$integration with respect to the kinematic singularities in the (forward) anti-parton - hadron scattering amplitude for the case of (non-vanishing) distribution functions for quarks (a) and antiquarks (b).

The forward amplitude itself has singularities (cuts) for positive parton virtuality $k^{2}$ and in the Mandelstam variables, for which we choose $s=(P-k)^{2}$ and $u=(P+$ $k)^{2}$. For the forward amplitude, the invariants are constrained to $s+u=2 k^{2}+2 M^{2}$, where we will neglect the hadron masses (they don't play any essential role in our proof). These singularities then constitute the $s$-cut (for $s>0$ and $u<0$ ) and the $u$-cut (for $u>0$ and $s<0$ ). Using the expansion for $k$ in Eq. (2), one has $k^{2}=2 x k^{-}+k_{T}^{2}$ or $s=2(x-1) k^{-}+k_{T}^{2}$ and a similar expression for $u$. The transverse momenta, just as any of the parton or hadron masses, have little bearing on our results so we omit them in the expressions for $k^{-}$,

$$
k^{-}=\frac{s+i \epsilon}{2(x-1)}=\frac{u+i \epsilon}{2(x+1)}=\frac{k^{2}+i \epsilon}{2 x},
$$

and one sees that for distribution functions the $k^{-}$integration with respect to $s, u$ and $k^{2}$ singularities follows the (dashed) contour in Fig. 3. The integration contours can be wrapped around the $s$ and $u$-cuts for positive and negative $x$-values respectively if $|x|<1$, cuts that (in $k_{1}^{-}$) smoothly vanish when $|x| \rightarrow 1$. Neither masses, nor transverse momenta matter and the support properties are valid for collinear and TMD PDFs [15]. We get

$$
\Phi(x)=\theta(x) \theta(1-x) \operatorname{Disc}_{[s]} \mathcal{A}+\theta(-x) \theta(1+x) \operatorname{Disc}_{[u]} \mathcal{A} .
$$

As discussed for instance in Ref. [32], the case for fragmentation is different since one in essence discusses the parton propagator for positive $k^{2}$ (sitting on the cut). 


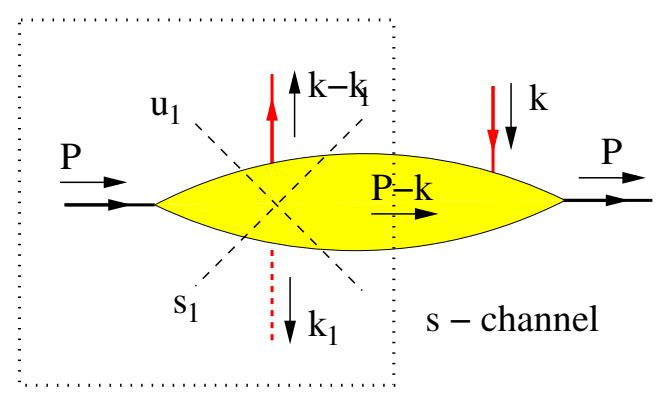

(a)

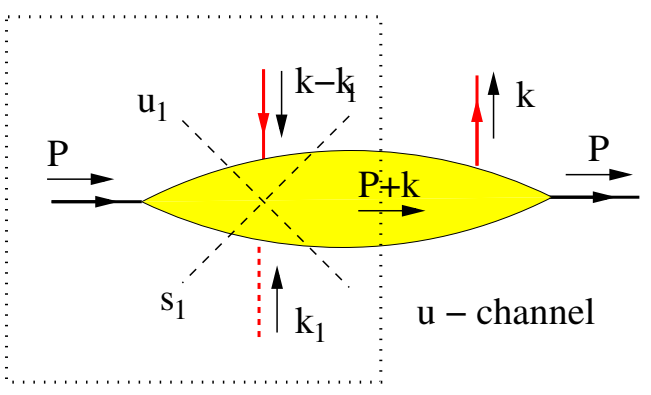

(b)

Fig. 4. The additional invariants for the amplitude $\mathcal{A}\left(k^{2} ; s, u ; s_{1}, u_{1} ; k_{1}^{2},\left(k-k_{1}\right)^{2}\right)$ relevant for gluonic pole matrix elements, (a) for the case $s>0$ and (b) for the case $u>0$.

For $|x|>1$, one simply has

$$
\Delta(x)=\theta(x-1) \operatorname{Disc}_{[s]} \mathcal{A}+\theta(-1-x) \operatorname{Disc}_{[u]} \mathcal{A} .
$$

In wrapping the integration around the $s$ - or $u$-cut we have to assume convergence in the variable $k^{-}$(or $k^{2}$ ), or use subtracted relations.

It is important to mention here that the integration of Eq. (1) and $\mathcal{A}\left(k^{2} ; s, u\right)$ over $k_{T}$ leads to ultraviolet divergences $[29,30,27]$. However, this does not invalidate the assumption that the integral over $k^{-}$alone is sufficiently well behaved when $k^{+}$and $k_{T}$ are fixed. Integration over $k_{T}$ as well as weighting with $k_{T}$ is anyway intimately linked with QCD evolution of TMDs [33].

\section{Analytic Structure for Multi-Partonic Correlations Functions}

We extend this analyticity analysis to the multi-parton distribution and fragmentation functions in Eq. (6), by looking at the multi-parton amplitude $\mathcal{A}\left(k^{2} ; s, u ; s_{1}, u_{1} ; k_{1}^{2},\left(k-k_{1}\right)^{2}\right)$ shown in Fig. 4, by studying the contours for the additional integrations 15 . Defining the momentum of the additional parton as $k_{1}$ as shown in Fig. 1, one retains the definitions and relations for $s$ and $u$. For given positive $s(s$-channel, $x>0)$ or positive $u(u$-channel, $x<0)$ one gets additional 


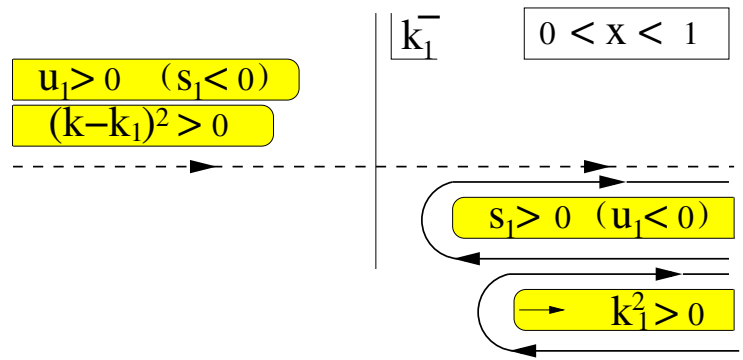

(a) $\Phi_{G}(x, x)$

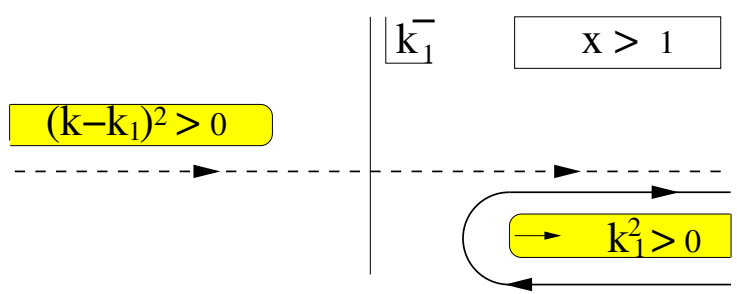

(b) $\Delta_{G}(x, x)$

Fig. 5. The integration contours for the $k_{1}^{-}$integration with respect to the singularities in the amplitude $\mathcal{A}\left(k^{2} ; s, u ; s_{1}, u_{1} ; k_{1}^{2},\left(k-k_{1}\right)^{2}\right)$ relevant for gluonic pole contributions. The figure shows for a given value of positive $s$ (relevant for $x>0$ ) how the $k_{1}^{-}$integration bypasses the cuts in $s_{1}, u_{1}$ and the parton virtualities in the limit $x_{1} \rightarrow+0$. The cases $0<x<1$ (distributions) and $x>1$ (fragmentation) are shown in Figs (a) and (b) respectively. In the latter case only parton virtualities are relevant.

invariants $s_{1}=\left(P \mp k \pm k_{1}\right)^{2}$ and $u_{1}=\left(P \mp k_{1}\right)^{2}$ (cf. Figs 4 (a) and (b), respectively). Note that $t_{1}=k^{2}$ in both cases. Furthermore one has parton virtualities. Depending on if one is dealing with the $s$ - or $u$-cut discontinuities, one has slightly different constraints for $s_{1}+u_{1}$, but for given values of $s$ and $u$ in the two cases of Fig. 4, one has cuts along $s_{1}>0\left(u_{1}<0\right)$ and $u_{1}>0\left(s_{1}<0\right)$ as well as for positive parton virtualities. The relevant singularities for $k_{1}^{-}$are found from

$$
\begin{aligned}
k_{1}^{-} & =\frac{s_{1}+i \epsilon}{2\left(x_{1}-(x \mp 1)\right)}+k^{-}=\frac{u_{1}+i \epsilon}{2\left(x_{1} \mp 1\right)} \\
& =\frac{k_{1}^{2}+i \epsilon}{2 x_{1}}=\frac{\left(k-k_{1}\right)^{2}+i \epsilon}{2\left(x_{1}-x\right)}+k^{-}
\end{aligned}
$$

(with $\mp$ referring to $s$ - and $u$-channel cuts, respectively). Again, parton or hadron masses as well as transverse momenta have little bearing as all they do is move the endpoints of the cuts. However, it is important to note that $k_{1 T}$ in the complete expression for the numerator of Eq. (10) protects against the cut starting at zero in the zero mass limit. Depending on the value of $x_{1}$, the integration contour in $k_{1}^{-}$bypasses the singularities encountered in the complex plane in a particular way, which dictates the support properties of the quark-gluon-quark correlation functions. The denominators in Eq. (10) in the expressions relating $k_{1}^{-}$to $s_{1}$ and $u_{1}$ tell 
us that only when $x_{1} \in[x-1,1]$ (for positive $x$ ) or $x_{1} \in[-1, x+1]$ (for negative $x)$ the singularities in $s_{1}$ and $u_{1}$ are relevant. We study the case of the $s$-channel $(x>0)$. The $u$-channel is analogous. Looking at gluonic poles, we consider the limit $x_{1} \rightarrow 0$. For $0<x<1$, the value $x_{1}=0$ lies in the interval for which the $s_{1}$ and $u_{1}$ discontinuities can contribute. These are shown in Fig. 5(a), now together with the singularities arising from the parton virtualities $k_{1}^{2}$ and $\left(k-k_{1}\right)^{2}$. For the case $x>1$ only these parton virtualities matter, shown in Fig. 5(b). We then find (including for $\Phi_{G}$ also the $u$-channel contribution) in the limit $x_{1} \rightarrow 0$

$$
\begin{aligned}
& \Phi_{G}(x, x)=\theta(x) \theta(1-x) \operatorname{Disc}_{\left[s, s_{1}\right]} \mathcal{A}+\theta(-x) \theta(1+x) \operatorname{Disc}_{\left[u, u_{1}\right]} \mathcal{A} \\
& \Delta_{G}(x, x)=0
\end{aligned}
$$

where for $\Delta_{G}(x, x)$ the $k_{1}^{-}$integration can be wrapped around the $k_{1}^{2}$ cut, which smoothly vanishes for $x_{1} \rightarrow+0$. This is described by the arrow inside the branch cut in Figs. 5(a) and (b), indicating that it harmlessly recedes to infinity. Moreover it matches continuously to the case that $x_{1}<0$. Starting from $x_{1} \rightarrow-0$ one immediately would have obtained Eqs. (11) and (12), since the $k_{1}^{2}$ cut is then along the negative $k_{1}^{-}$axis. This establishes the proof that gluonic pole matrix elements for fragmentation correlators vanish. Similar results were obtained in the spectator model field theory in Ref. [17], the spectator approach in Ref. [18], and the general spectral approach of Ref. [19]. But now it has been demonstrated in a completely general way by assuming unitarity and analyticity properties to hold for QCD. Similar to the earlier discussion, we note that the integration of Eq. (6) and $\mathcal{A}\left(k^{2} ; s, u ; s_{1}, u_{1} ; k_{1}^{2},\left(k-k_{1}\right)^{2}\right)$ over $k_{1 T}$ is ultraviolet divergent $[29,30,27]$. Again, the integral over $k_{1}^{-}$alone is well behaved when $k_{1}^{+}$and $k_{1 T}$ are fixed with again full integration over $k_{1 T}$ corresponding to appropriate regularization of $\mathcal{A}\left(k^{2} ; s, u ; s_{1}, u_{1} ; k_{1}^{2},\left(k-k_{1}\right)^{2}\right)$ [34 ] and study of the QCD evolution [33].

In our last step, we show that our arguments for vanishing gluonic pole matrix elements hold for general multi-gluonic and even multi-partonic pole matrix elements. Considering the analytic properties of general multi-gluonic pole matrix elements we can proceed inductively. For two gluons one simply extends the nesting of momenta $k-k_{1}$ and $k_{1}$ by a nesting $k-k_{1}-k_{2}, k_{1}-k_{2}$ and $k_{2}$, which adds to the set $\left(s, u, s_{1}, u_{1}\right)$ two new invariants $\left(s_{2}, u_{2}\right)$, without changing the behavior in the others. The gluonic pole matrix element $\Delta_{G G}(x, x, x)$ thus disappears as do all higher pole matrix elements. Since these higher pole matrix elements appear in the higher $k_{T}$-moments of the correlator $\Delta_{i j}^{[\mathcal{U}]}\left(z, k_{T}\right)$ in Eq. (4), we conclude based on our very general assumptions of analyticity for QCD amplitudes that this TMD correlator is universal and will be convoluted with the standard partonic cross sections. There is no proliferation of functions originating from the structure of the gauge links [14]. This universality thus applies to all TMD fragmentation functions, T-even or T-odd, and for quark as well as for gluon PFFs. Most well-known are the T-odd ones for quarks such as the Collins function $H_{1}^{\perp}[16,4]$ and the polarization fragmentation function $D_{1 T}^{\perp}[4,35]$. These functions are simply allowed T-odd 
parts in the fragmentation correlator $\Delta\left(z, k_{T}\right)$ being a decay function. The corresponding T-odd TMD distribution functions, the Boer-Mulders function $h_{1}^{\perp}$ and the Sivers function $f_{1 T}^{\perp}$ originate from the difference $\Phi^{\left[\mathcal{U}_{+}\right]}\left(x, p_{T}\right)-\Phi^{\left[\mathcal{U}_{-}\right]}\left(x, p_{T}\right)$ of correlators with different gauge links and as a consequence will be convoluted with non-standard gluonic pole cross sections $[13,14,36]$. Our result also implies universality for the TMD fragmentation functions of gluons [37]; including for instance the T-even TMD fragmentation functions $H_{1}^{\perp(g)}$, which just as the corresponding distribution function $h_{1}^{\perp(g)}$ has a non-trivial gauge link dependence [36, 38, 39]. The T-even fragmentation functions $H_{1}^{\perp(g)}$, however, is universal. In the case of these T-even functions, the non-trivial gauge link dependence only becomes visible in even $k_{T}$-moments involving contributions from T-even multi-gluonic pole matrix elements with an even number of gluons, all of which for fragmentation functions, however, will vanish.

\section{Acknowledgments}

LG thanks the organizers of the QCD Evolution Workshop for their invitation to present this work, and for their hospitality. LG acknowledges support from U.S. Department of Energy under contract DE-FG02-07ER41460.

\section{References}

1. J. C. Collins and D. E. Soper, Nucl. Phys. B194, 445 (1982).

2. R. L. Jaffe and X.-D. Ji, Phys. Rev. Lett. 67, 552 (1991).

3. P. J. Mulders and R. D. Tangerman, Nucl. Phys. B461, 197 (1996).

4. D. Boer and P. J. Mulders, Phys. Rev. D57, 5780 (1998).

5. A. V. Efremov and O. V. Teryaev, Sov. J. Nucl. Phys. 36, 140 (1982).

6. A. V. Efremov and O. V. Teryaev, Phys. Lett. B150, 383 (1985).

7. J.-W. Qiu and G. Sterman, Phys. Rev. Lett. 67, 2264 (1991).

8. J.-W. Qiu and G. Sterman, Nucl. Phys. B378, 52 (1992).

9. J.-W. Qiu and G. Sterman, Phys. Rev. D59, 014004 (1999).

10. Y. Kanazawa and Y. Koike, Phys. Lett. B478, 121 (2000).

11. H. Eguchi, Y. Koike and K. Tanaka, Nucl. Phys. B763, 198 (2007).

12. Y. Koike and K. Tanaka, Phys. Lett. B646, 232 (2007).

13. A. Bacchetta, C. J. Bomhof, P. J. Mulders and F. Pijlman, Phys. Rev. D72, 034030 (2005).

14. C. J. Bomhof and P. J. Mulders, JHEP 02, 029 (2007).

15. L. P. Gamberg, A. Mukherjee and P. J. Mulders, Phys. Rev. D83, 071503 (2011).

16. J. C. Collins, Nucl. Phys. B396, 161 (1993).

17. J. C. Collins and A. Metz, Phys. Rev. Lett. 93, 252001 (2004).

18. L. P. Gamberg, A. Mukherjee and P. J. Mulders, Phys. Rev. D77, 114026 (2008).

19. S. Meissner and A. Metz, Phys. Rev. Lett. 102, 172003 (2009).

20. A. V. Efremov and A. V. Radyushkin, Theor. Math. Phys. 44, 774 (1981).

21. D. Boer and P. J. Mulders, Nucl. Phys. B569, 505 (2000).

22. A. V. Belitsky, X. Ji and F. Yuan, Nucl. Phys. B656, 165 (2003).

23. D. Boer, P. J. Mulders and F. Pijlman, Nucl. Phys. B667, 201 (2003).

24. J. C. Collins and D. E. Soper, Nucl. Phys. B193, 381 (1981). 
25. X.-d. Ji, J.-p. Ma and F. Yuan, Phys. Rev. D71, 034005 (2005).

26. J. Collins, T. Rogers and A. Stasto, Phys. Rev. D77, 085009 (2008).

27. A. Bacchetta, D. Boer, M. Diehl and P. J. Mulders, JHEP 08, 023 (2008).

28. P. V. Landshoff and J. C. Polkinghorne, Phys. Rept. 5, 1 (1972).

29. R. L. Jaffe, Nucl. Phys. B229, 205 (1983).

30. M. Diehl and T. Gousset, Phys. Lett. B428, 359 (1998).

31. C. Itzykson and J. B. Zuber, QUANTUM FIELD THEORY (1980), New York, USA: Mcgraw-hill (1980) 705 P.(International Series In Pure and Applied Physics).

32. J. C. Polkinghorne, MODELS OF HIGH-ENERGY PROCESSES (1980), Cambridge, UK: Univ.Pr.(1980) 131p.

33. S. Aybat and T. C. Rogers, Phys. Rev. D83, 114042 (2011).

34. Z.-B. Kang, Phys. Rev. D83, 036006 (2011).

35. D. Boer, Z.-B. Kang, W. Vogelsang and F. Yuan, Phys. Rev. Lett. 105, 202001 (2010).

36. C. Bomhof and P. J. Mulders, Nucl. Phys. B795, 409 (2008).

37. P. J. Mulders and J. Rodrigues, Phys. Rev. D63, 094021 (2001).

38. F. Dominguez, C. Marquet, B.-W. Xiao and F. Yuan, Phys. Rev. D83, 105005 (2011).

39. D. Boer, S. J. Brodsky, P. J. Mulders and C. Pisano, Phys. Rev. Lett. 106, 132001 (2011). 